\title{
On the Efficiency of Secure Beaconing in VANETs
}

\author{
Elmar Schoch \\ Ulm University \\ Ulm, Germany \\ elmar.schoch@uni-ulm.de
}

\author{
Frank Kargl \\ University of Twente \\ Enschede, The Netherlands \\ f.kargl@utwente.nl
}

\begin{abstract}
Direct inter-vehicle communication enables numerous safety applications like intersection collision warning. Beacons periodic one-hop link-layer broadcast messages containing, e.g., location, heading, and speed - are the basis for many such applications. For security, current work often requires all messages to be signed and to carry a certificate to ensure integrity and authenticity. However, high beacon frequency of $1-10 \mathrm{~Hz}$ and dense traffic situations lead to significant communication and computational overhead. In this paper, we propose several mechanisms to significantly reduce this overhead while maintaining a comparable level of security. The general idea is to omit signatures, certificates, or certificate verification in situations where they are not necessarily required. This creates a security-performance trade-off that we analyze in detail. The results show that significant savings can be achieved with only small impact on security.
\end{abstract}

\section{Categories and Subject Descriptors}

C.2.0 [Computer-Communication Networks]: General

\section{General Terms}

Security, Reliability, Performance

\section{Keywords}

Vehicular ad hoc networks (VANETs), Security, Efficiency

\section{INTRODUCTION}

When looking at recent standardization efforts and fields tests it becomes clear that beaconing will initially be the most important form of communication for upcoming C2X eSafety applications. Examples of such applications include all forms of cooperative awareness messages like intersection collision warning where vehicles approaching an intersection periodically exchange status data to detect potential collisions and warn drivers accordingly.

Permission to make digital or hard copies of all or part of this work for personal or classroom use is granted without fee provided that copies are not made or distributed for profit or commercial advantage and that copies bear this notice and the full citation on the first page. To copy otherwise, to republish, to post on servers or to redistribute to lists, requires prior specific permission and/or a fee.

WiSec'10, March 22-24, 2010, Hoboken, New Jersey, USA.

Copyright 2010 ACM 978-1-60558-923-7/10/03 ...\$10.00.
In the context of vehicular communication, beaconing is the periodic transmission of packets as (single-hop) linklayer broadcast to nearby vehicles or road-side units [1]. Those packets typically contain at least the current location, heading, and speed of the sending vehicle.

Despite its benefits, inter-vehicle communication (IVC) systems also open opportunities for abuse when implemented in naive and insecure ways. Security mechanisms are needed, which ensure integrity and that a receiver is able to recognize valid senders. Many solutions use signatures based on asymmetric cryptographic mechanisms like ECDSA together with other mechanisms [2, 3].

On the other hand, signatures and certificates lead to a significant consumption of communication bandwidth and processing power. The earlier aggravates the problem that the wireless communication medium can become oversaturated in situations of high vehicle density, the latter demands considerable CPU performance.

The goal of this work is to provide a practically-oriented solution that reduces this security overhead without substantially reducing security.

\section{ANALYSIS AND RELATED WORK}

When beaconing is unprotected, internal and external attackers can easily run attacks like spoofing, beacon suppression, manipulation, or replaying. Evidently, there is a strong need to prevent especially those attacks that might negatively affect reliability of eSafety applications. Integrity protection can prevent spoofing and manipulation attacks. Combined with timestamps or serial numbers this also addresses replay attacks. We do not consider beacon suppression here. In general, most security mechanisms proposed so far apply the following basic approach $[2,3]$ :

1. Vehicles are equipped with asymmetric cryptographic key pairs $(V K, S K)$ and certificates (Cert) issued by a trusted certification authority $(C A)$.

2. Senders sign beacons using the vehicle's signature key $S K$, and receivers verify them using the corresponding verification key $V K$. Both signature and certificate containing $V K$ are attached to every beacon.

3. Beacons not containing a valid signature and certificate are ignored by receivers.

4. Beacons contain timestamps or sequence numbers to prevent replay attacks.

5. Signature keys are stored in and managed by tamperresistant hardware to prevent extraction from vehicles for unauthorized use.

Systems following the outlined approach have been pro- 


\begin{tabular}{|r|c|c|}
\hline Neighbors & 30 byte payload & 211 byte payload \\
\hline 1 & 4901,96 & 2225,52 \\
\hline 50 & 98,04 & 44,51 \\
\hline 100 & 49,02 & 22,26 \\
\hline 250 & 19,61 & 8,90 \\
\hline 500 & 9,80 & 4,45 \\
\hline
\end{tabular}

Table 1: Packet rates per node per second

posed e.g. in the IEEE 1609.2 standard, the SeVeCom and NoW projects, and by various other researchers. Variations include the use of pseudonyms for privacy protection, the certification architecture, or the use of group signatures [4]. But all approaches introduce two significant problems:

1. Signatures and certificates increase the beacons' size and create a significant protocol overhead.

2. Creating and verifying signatures creates a significant computational overhead.

Most approaches suggest the use of Elliptic Curve Cryptography (ECC) in vehicular networks, as ECC signatures, keys, and certificates are smaller than their RSA counterparts [5]. A 224-bit ECDSA signature excluding certificate requires 56 bytes compared to 256 bytes for an equivalent RSA-2048 signature. Including certificates and additional management information, a 224-bit ECDSA signature and certificate as proposed by IEEE 1609.2 [3] needs 181 bytes, a corresponding RSA-2048 certificate about $3-4$ times that size. In accordance with [3], we assume 56 bytes for the signature and 125 bytes for the certificate in this paper.

Using ECC instead of RSA lessens problem 1 to some extent, but signature plus certificate still require a substantial amount of bytes. Whereas ECC signature generation is fast, verification is comparatively costly, so problem 2 remains. Use of dedicated ECC ASICs [6] would increase manufacturing costs.

[7] builds on TESLA with delayed key disclosure using message authentication codes (MACs) instead of costly asymmetric cryptography. However, the approach requires strict time synchronization and allows only delayed beacon verification after key disclosure. This does not fit well to dynamic and time-critical vehicular networks.

A deeper analysis of the impact of additional 181 bytes payload is provided in Tab.1. The table shows the theoretical upper bound of packets that can be sent per node and per second for different node densities. The values were calculated using the equation

$$
T X=T_{P R E}+T_{S I G}+\frac{(16+8 * L E N G T H+6)}{D A T A R A T E}+\frac{T_{S Y M}}{2}
$$

from [8], assuming $6 \mathrm{Mbps}$ data rate, $T_{P R E}=32 \mu \mathrm{s}, T_{S I G}=$ $T_{S Y M}=8 \mu \mathrm{s}$, a 36 octets MAC header, and AC-3 traffic [9].

Although realistic values will be lower due to packet collisions, one can easily see that in high vehicle densities above 250 neighbors, $10 \mathrm{~Hz}$ beaconing rate cannot be sustained any more with 211 bytes payload. Considering only 30 bytes sized packets without security overhead, almost 500 neighbors can be supported.

Computational overhead of signing beacons is one signature generation when sending a beacon sent, and up to two signature verifications, when receiving a beacon; verifying the packet signature and the $\mathrm{CA}$ signature of the certificate. Assuming a neighbor density of 200 vehicles and beaconing rates between 1 and $10 \mathrm{~Hz}$, each vehicle needs to generate between 1 and 10 signatures per second and

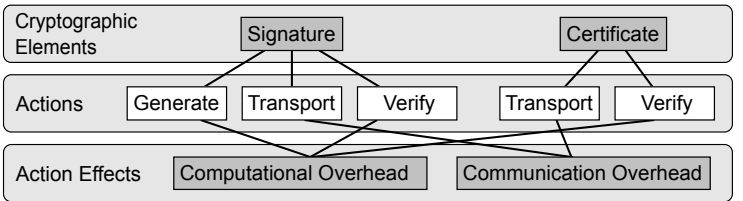

Figure 1: Overhead due to signing.

needs to verify between 400 and 4000 signatures per second. Typical hardware for on-board units used in upcoming fieldtrials is expected to have e.g. a Power PC CPU at 400 $\mathrm{MHz}$ [10]. Actual crypto performance depends very much on implementation, but own experiments indicate that this hardware will not be able to do more than a few dozens of verifications per second using ECDSA-224 and optimized software libraries. Dedicated ASICs are expected to be able to handle the cryptographic load at moderate costs [6].

Still, additional costs for ASICs and scarce channel bandwidth motivates our work on mechanisms to reduce computational and protocol overhead of signed beacons while maintaining at least a comparable level of assurance.

\section{EFFICIENT SECURE BEACONING STRATEGIES}

As outlined earlier, signature based protection of beaconing involves signatures as well as certificates. Fig. 1 illustrates actions and related overhead. Signatures need to be generated, transported, and verified, whereas certificates are pre-generated and only need to be transported and verified. Generation and verification lead to computational overhead, transport to communication overhead.

Aiming at the reduction of protocol and computational overhead, one could basically

- omit beacon signature generation,

- omit transmitting a certificate with a message,

- omit transmitting a signature with the message,

- omit certificate verification, or

- omit beacon signature verification.

Multiple of these options can be applied simultaneously with certain dependencies, where e.g. omitting the certificate also implies omitting the certificate verification. While we roughly sketch some of the ideas presented here in [11] and $[4,12]$ proposes a basic form of certificate omission, this is the first work to comprehensively discuss all the different alternatives. We also propose several advanced omission strategies and analyze their consequences in terms of protocol and computational complexity, and particularly in terms of security implications as some beacons will not be verifiable instantly after reception. Our strategies do not omit operations unconditionally, but instead only in certain cases depending on a vehicle's context or with a certain probability. This allows a fine-grained tuning of a securityperformance trade-off. We argue that such a probabilistic approach to security could be an interesting alternative to classic deterministic approaches that try to deliver security guarantees at a high cost. Probabilistic security strategies might fit very well to dynamic networks like VANETs.

\subsection{Omitting Certificates and Certificate Ver- ifications}

A substantial part of security-induced communication overhead is the certificate attached to every message. A 
certificate of the sender is needed by a receiving node for two reasons: First, the certificate contains the verification key $V K$ needed to verify the signature. Second, the certificate asserts validity of $V K$ by a trusted authority.

As already proposed in $[4,12,11]$, one can reduce computational overhead of certificate verifications without negative effect by storing verified certificate signatures in a local signature store. This saves the costly verification of certificates attached to subsequent packets from the same node, and thus already cuts the computational costs of handling received packets almost by half.

We can even reduce communication overhead notably by storing certificates: If node $A$ locally caches a verified certificate of another node $B$, then $B$ can omit its certificate in subsequent packets. If signature and ID contained in a beacon allow identification of the corresponding $V K$, a receiving node can verify the signature of received beacons without certificate. The only question is, when must a node attach his certificate, and when may he omit it? If communication partners have not exchanged certificates previously, omission of certificates will lead to cases where a node receives a beacon containing no certificate from a node from which it has no certificate yet. Such a packet must be regarded as invalid or signature verification must be delayed until $V K$ is available. As the amount of bandwidth that can be saved by omitting 125 bytes certificates is tremendous, we argue that certificate omission is reasonable if the time period until a node is able to verify such beacon signatures is small and this case does not occur too often.

$[4,12]$ proposes to reduce communication overhead by leaving out certificates on a periodic schedule, i.e., only every $n^{t h}$ beacon packet contains a certificate. We call this Periodic Omission of Certificates (POoC) here. Assuming periodic beaconing with a beacon interval $\Delta b$, the period until a node can verify packets from another node is $(n-$ 1) $\cdot \Delta b$ in the worst case. While the scheme reduces the communication overhead, it has the drawback that it is independent of vehicle context. For instance, if a vehicle drives fast, the consequence of providing a certificate only every $n^{\text {th }}$ packet may result in violation of safety margins. With $\Delta b=0.5 \mathrm{~s}$ and $n=4$, a vehicle may have to wait up to 1.5 seconds until it can verify the beacons of another vehicle. At $200 \mathrm{~km} / \mathrm{h}$, this corresponds to 83 meters, too much for many safety applications. With higher beacon frequency and lower speed, the scheme may be appropriate, though.

We propose an enhanced neighbor-based scheme for certificate omission that takes into account topology changes explicitly and respects the beacon interval length.

\subsubsection{Neighbor-based Certificate Omission ( $\mathrm{NbCO}$ )}

Here, we utilize the fact that every node roughly learns its neighbors in wireless transmission range through beaconing. A node can monitor neighborhood changes and base the decision whether to attach certificates or not.

When node $A$ is about to send a beacon $b_{t_{i+1}}, A$ determines if new neighbors were added to its neighbor table since the last beacon $b_{t_{i}}$. If yes, a certificate $\operatorname{Cert}(V K)$ is attached to beacon $b_{t_{i+1}}$, else it is sent without certificate.

This leads to cases where a node cannot verify a beacon because it does not yet possess an appropriate certificate. However, the worst case is to wait for one beacon interval period because the receiving node will include its certificate in the subsequent beacon following the described strategy.
Yet, independent of the omission scheme, more certificate misses may occur due to a lossy channel. In order to avoid indeterminably long wait times for the certificate, we propose that nodes could explicitly solicit for certificates if a certificate is not available within $\Delta b$. In the example, $B$ would send a certificate request causing $A$ to include a certificate in the next beacon. Alternatively, nodes could send certificates in $n$ consecutive beacons after a neighborhood change to reduce the chance for packet loss.

As such modifications might make our results highly dependent on channel loss assumptions, we will not consider such enhancements for the moment and just implement the basic approach in our evaluation where we show the effectiveness and suitability both regarding saved bandwidth and unverifiable beacons.

\subsection{Omitting Signatures and Sig. Verification}

Additional communication overhead is saved by skipping signatures entirely. This implies also that signatures do not have to be created and verified, avoiding any security overhead. However, omitting signatures also nullifies authentication and integrity protection - which means that the data should not be used for any critical applications. Following the general idea of this work, we propose to use signatures selectively, that is, to secure only a certain share of all beacons depending on the situation. This saves overhead where possible, but also maintains security where necessary.

\subsubsection{Situation-based Signing}

As a baseline, every $n^{t h}$ beacon or one beacon in a time interval $t$ should be signed to ensure a continuous chain of trusted data. Unsecured data is sent in between, which can be matched with the trusted data for example using movement prediction. Although this saves overhead, critical situations require more trustworthiness to enable reliable decisions for safety-related applications. Therefore, we propose situation-based signing to balance between security and communication overhead.

In this scheme, we base the decision to sign a beacon or not on current vehicle context. By default, all beacons are only periodically signed. As soon as a vehicle discovers a potentially dangerous situation using the insecure beacons, it starts to attach signatures to all sent beacons. Assuming that all involved vehicles detect hazards independently, all sent beacons will be signed in critical situations.

\subsubsection{Omitting Signature Verification}

Computational load is more or less exclusively determined by signature verifications and not by signature generations. This is mostly because a vehicle will receive a magnitude more packets than it sends in dense traffic and will thus have to do much more signature verifications than generations.

When addressing computational load, it is therefore reasonable to save on signature verifications and let the receiver decide which signatures to verify and which not. If a vehicle decides which signatures should be verified and which can be left unchecked, the receiver can directly control its computational load. The drawback is of course that an attacker might inject spoofed packets with invalid signatures hoping that receivers will not check them. Hence the goal is to minimize such missed false signatures, while effectively reducing the computational load. 


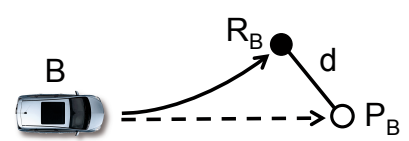

Figure 2: Calculation of position prediction error $d$

Periodic Verification Strategy: Here, each vehicle always verifies signatures of beacons of new vehicles not yet contained in its neighbor table. From that on, it only verifies every $n^{\text {th }}$ signature from the same source. Intermediate beacons are accepted as genuine without further checking. Without mobility and resulting neighbor changes, this strategy reduces the verification rate down to $\frac{1}{n}$.

As indicated, an attacker might create packets using the spoofed source address of other neighboring vehicles and invalid signatures and hope that other vehicles accept these packets. Chances that packets are accepted by another vehicle are pretty good, actually $\frac{n-1}{n}$. In those beacons, the attacker can claim arbitrary positions, vehicle speeds, etc. Assuming that damage done by false information corresponds to the deviation from real values, our second strategy takes this into account and verifies only those packets that contain suspicious values.

Context-Adaptive Verification Strategy: Here, we predict upcoming beacon information from neighboring vehicles. If vehicle $A$ has received a series of beacons containing position and speed from vehicle $B, A$ can likely predict future position and speed of $B$ using a linear estimation like a Kalman filter. The Kalman filter is an efficient recursive filter that estimates the state of a dynamic system from a series of incomplete and noisy measurements.

As before, $A$ verifies the signature of the initial packet from $B$ as well as every $n^{t h}$ packet. $n$ can be selected comparatively large. $A$ also initializes a new Kalman filter $K_{B}$ for $B$ s position and speed. As illustrated in Fig. 2, whenever a new beacon from $B$ is received, $A$ updates the Kalman filter and calculates the position prediction error $d$, i.e., the distance between prediction $P_{B}$ and $R_{B}$ contained in the beacon. The probability $p$ to verify the beacon's signature is chosen based on $d$.

The deviations from the likely behavior of another vehicle should determine the risk for spoofed packets to be checked. We set the signature verification probability $p_{\text {check }}$ as

$$
p_{\text {check }}(d)=\omega *\left(1-e^{-(d / \beta)^{\alpha}}\right)
$$

This is the cumulative distribution function of a Weibull distribution multiplied by a weight $\omega$. A detailed discussion of this is provided in the evaluation section (Section 4.2). Further optimizations of the scheme could verify beacons of risky positions first and take the currently available computational resources into account.

\section{EVALUATION}

We now analyze some aspects of the mentioned approaches by means of simulations. After introducing our simulation environment, we analyze the efficiency and security of certificate omission as well as of signature verification omissions. We have chosen these two examples to show the potential of our approaches to significantly reduce overhead while keeping the actual level of security.

In order to evaluate the effectiveness of our proposed schemes, we conducted simulations using the Java-based simulation tool JiST/SWANS [15]. Based on version 1.0.6,

\begin{tabular}{|c|c|c|}
\hline Parameter & Urban & Highway \\
\hline Number of nodes & $100-1000$ & $\approx 100-1000$ \\
\hline Field size & $\begin{array}{l}\approx 3000 \mathrm{~m} \times \\
3000 \mathrm{~m}\end{array}$ & $\begin{array}{l}\approx 12 \mathrm{~km}, \text { mult. } \\
\text { lanes per dir. }\end{array}$ \\
\hline Node dens. (neigh./node) & \multicolumn{2}{|c|}{$\approx 5-22$} \\
\hline Node velocity $(\mathrm{m} / \mathrm{s})$ & $\approx 25$ & $\approx 40$ \\
\hline Mobility model & STRAW [13] & FleetNet $[14]$ \\
\hline Link-/MAC-Layer & \multicolumn{2}{|c|}{ IEEE $802.11 \mathrm{p}, 5.9 \mathrm{GHz}, 3 \mathrm{MBit} / \mathrm{s}$} \\
\hline Transmission range $(\mathrm{m})$ & \multicolumn{2}{|r|}{250} \\
\hline Beacon interval (ms) & \multicolumn{2}{|c|}{$100-1000$} \\
\hline Simulation time $(\mathrm{s})$ & \multicolumn{2}{|r|}{60} \\
\hline Simulation runs & \multicolumn{2}{|r|}{10} \\
\hline
\end{tabular}

Table 2: Overview on simulation parameters

we added several extensions, particularly the beaconing mechanism including neighbor tables, a security module to virtually attach certificates and signatures, and an implementation of the Kalman filter to predict neighbor positions. Relevant simulation parameters are summarized in Tab. 2.

\subsection{Efficiency of $\mathrm{NbCO}$}

We first investigate the efficiency of neighbor-based certificate omission (NbCO, Section 3.1.1) and compare it with periodic omission (POoC) of certificates as presented in [4, 12]. We use two metrics for this evaluation. First, we measure the numbers of beacons with and without certificate attached. The percentage of beacons sent without certificate directly reflects the saved bandwidth as every certificate has a fixed size. Second, the number of not instantly verifiable beacons due to missing certificates is important because nodes then have to delay verification until they receive a corresponding certificate. While the neighborbased approach implicitly provides certificates with the next beacon, the amount of such cases still should be low.

Several factors influence the proposed schemes. One core factor is the beacon frequency. If beacons are transmitted with high frequency, saving bandwidth is particularly important to reduce channel load. Another influencing factor is node density $\rho$, which we denote as the average number of neighbors of a node. Saving bandwidth is more valuable in high-density scenarios, as the channel is more likely congested here. A third aspect is related to mobility in VANETs. Node movement patterns differ notably between cities and highways. In particular the highway scenario poses a special challenge to the system because nodes move fast, on multiple lanes, and on long road segments.

\subsubsection{Bandwidth Savings}

Fig. 3 (left) shows the amount of certificates that are omitted by $\mathrm{NbCO}$ and $\mathrm{POoC}$. In most cases, $\mathrm{NbCO}$ omits certificates in more than $70 \%$ of all sent beacons. One key influence is the time between consecutive beacons. The longer the beacon interval, the more beacons are sent with certificates attached and the less bandwidth can be saved. This is to be expected as the likelihood of neighborhood changes increases with the length of this period and certificates are only attached if the a new neighbor was discovered since the last beacon. In case of $10 \mathrm{~Hz}, \mathrm{NbCO}$ saves over $95 \%$ of bandwidth induced by certificate transfer. In contrast, in case of POoC and $n=3$, savings are independent of the beacon interval and stay at $66 \%$. Hence, the NbCO performs better in case of high and medium beaconing frequency, whereas $\mathrm{POoC}$ is better with larger beacon intervals. Results in Fig. 3 (left) also show 

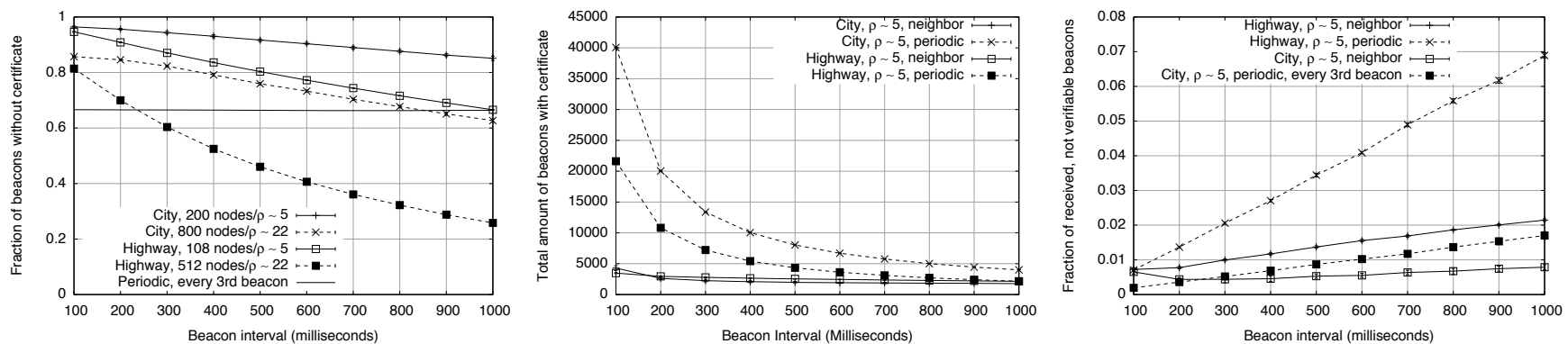

Figure 3: Certificate Omission: Relative saving (left), absolute saving (middle), unverifiable packets (right)

the influence of node movement patterns and node densities. High node velocity and traffic in only two directions lead to more neighborhood changes and thus less effectiveness on highways. The same applies to situations with higher node density. Comparing low density of $\rho \approx 5$ neighbors with a medium density of $\rho \approx 22$ neighbors per node, NbCO performs worse than the POoC under high node density, large topology change rate, and low beacon frequencies.

Looking at absolute numbers of sent beacons in Fig. 3 (middle), we find that for $\mathrm{NbCO}$ the absolute number of beacons sent with certificate attached is almost constant regardless of the beacon interval because the number of beacons with attached certificate is governed exclusively by topology changes. This contrasts to POoC that highly depends on the beacon rate. This is particularly relevant because the overall certificate overhead can be kept constant with respect to the beacon interval. Especially with high frequency and thus increased channel capacity problems, the neighbor-based approach yields best results.

\subsubsection{Unverifiable Packets}

As we explicitly accept the case that a node may not be able to verify a packet instantly when omitting certificates, it is important to analyze this potential drawback. In the worst case, several missing position updates may lead to an accident because the driver could not be warned in time, rendering the application useless. Hence, as few as possible beacons should be unverifiable and the delay until verification should be as low as possible.

Fig. 3 (right) indicates, that in most scenarios, less than $2 \%$ of all received packets are affected by this problem. Again, the highway scenario is affected more severely due to more topology changes. Complementing the earlier findings, more beacons cannot be verified in scenarios with low node density - which is normal, because less beacons carry certificates in these scenarios as shown before.

In the periodic approach, notably more beacons cannot be verified instantly compared to the neighbor-based scheme. This is particularly visible in the highway scenario - where it is most important to be able to react quickly because of high node velocity. Moreover, the worst case delay to receive a beacon with certificate attached depends on $n$. While Fig. 3 (right) only shows results for an average vehicle density of $\rho \approx 5$, similar results could be reproduced with high density.

To finally compare the periodic omission and the neighborbased approach, we relate saved bandwidth and unverifiable beacons in an efficiency metric. We calculate

$$
\text { Efficiency }=\frac{\text { Beacons } w / o \text { Certificate }}{\text { Not instantly verifiable beacons }}
$$

As shown in Fig. 4, $\mathrm{NbCO}$ yields better efficiency in all

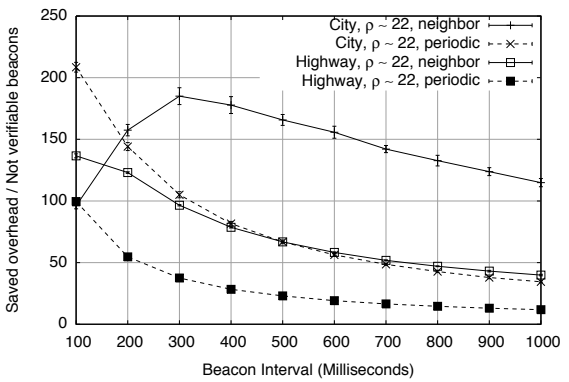

Figure 4: Protocol efficiency, saved bandwidth vs. unverifiable beacons.

cases except the city scenario with $10 \mathrm{~Hz}$ beacon frequency. Looking at the previous figures, the reason for this can be found in a certain "overoptimization" in this scenario. POoC achieves less unverifiable beacons because of a comparatively moderate reduction of the overhead.

In summary, $\mathrm{NbCO}$ achieves very low overhead with high beacon frequencies, constant overall certificate overhead independent of beacon interval, a maximum delay until verification of one beacon interval, and a generally low percentage of beacons that are not verifiable instantly.

\subsection{Efficiency of Sign. Verification Omissions}

Eq. 1 uses a cumulative Weibull distribution function as probability distribution function for verifying a packet signature. The base is the distribution of Kalman prediction errors, i.e., the distance by which the Kalman filter is off the correct positions, in a normal simulation. We determined this distribution and suitable parameters $(\alpha=4, \beta=10)$ by extensive simulation experiments in many scenarios.

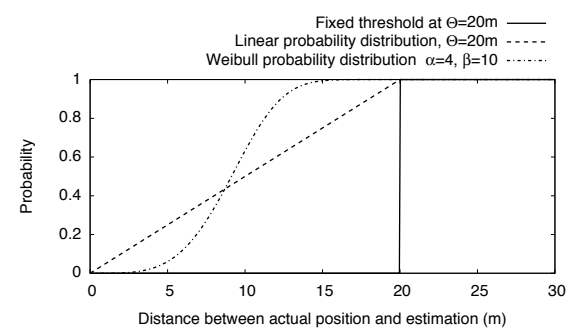

Figure 5: Different probability distributions.

To show the suitability of the cumulative Weibull distribution function, we compare it with two others shown in Fig. 5. One is a fixed threshold where a distance prediction error beyond $20 \mathrm{~m}$ leads to verification while packets with lower error are not checked. The second uses a linearly increasing probability between 0 and $20 \mathrm{~m}$.

In our simulations, we use attackers spoofing positions within a range of $200 \mathrm{~m}$ around their actual position. We 

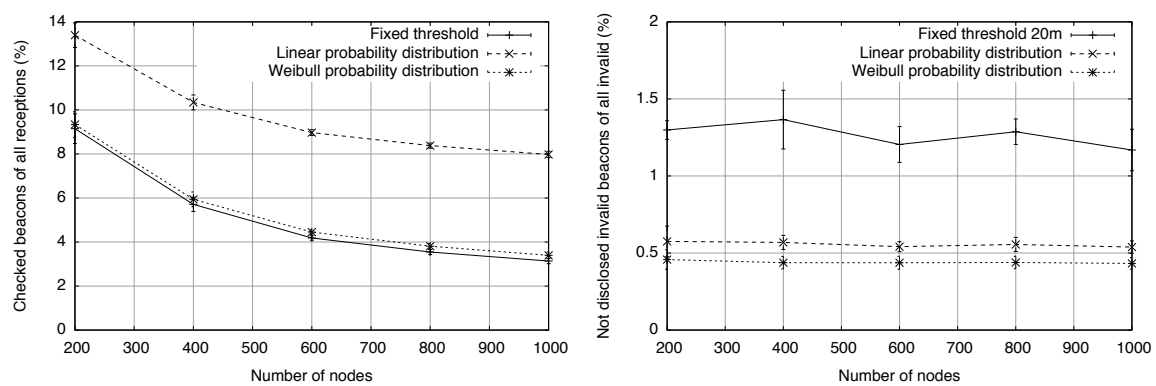

Figure 6: Signature verification omission: Packets checked (left) vs. unverified faked beacons (right)

argue that entirely random position claims can easily be detected by a maximum radio distance thresholds. Moreover, the distance between positions in consecutive beacons is limited to a maximum of $50 \mathrm{~m}$. As normal vehicles do not drive that fast, larger jumps can be assumed improbable within one beacon interval and thus be disclosed by a fixed threshold. Kalman filter settings were also determined by traffic simulations.

The results are shown in Fig. 6. On the left, one can see that only a small fraction of signatures are actually checked. For the linear distribution, this ranges between 8 and $14 \%$ while the fixed threshold and Weibull distribution give almost identical results between 3 and 10\%. This directly translates to reduced computational overhead, as every omitted signature verification saves one cryptographic operation. Savings are higher for denser networks because in less dense networks our mobility model makes vehicles move faster. This reduces the accuracy of the Kalman filter and thus increases the fraction of verified beacons.

While from a performance point of view, fixed threshold and Weibull distribution seem equivalent, the right graph shows that Weibull is actually superior. It shows the fraction of attacker beacon messages that were not verified by recipients. While with linear and Weibull distribution around $0.5 \%$ of attacker beacons were not detected, the fixed threshold misses around $1.25 \%$ of the attacker beacons. So using the cumulative Weibull distribution function for the signature verification decision provides a clear advantage over the other two strategies. Note that the percentage of missed attacker beacons is independent from node density.

Like with our previous evaluation, one can again conclude that applying the context-adaptive signature verification omission significantly reduces computational load at a bearable price. Missing $0.5 \%$ of attacker beacons gives an attacker only a very small chance of cheating.

\section{CONCLUSION}

The problem of communication and computational overhead due to security mechanisms is particularly distinctive for beaconing, because beacon packets are transmitted as broadcasts with comparatively high frequency by every vehicle. Generating signatures, transmitting signatures and certificates, and verifying them on the receiver side causes notable communication and computational overhead solely for the purpose of authenticity and integrity protection. While adding dedicated cryptographic hardware might solve the latter problem at a noticeable cost, at least the problem of network bandwidth requires additional solutions.

In this paper, we address this problem and propose schemes to reduce communication and computational over- head. The main concept of our approaches is to omit generation, transmission, and verification of signatures and certificates where this is possible without significant infringement of security. The analysis carried out has shown the efficiency of two of the proposed schemes and considers the level of security achieved compared to the case when all beacon packets are signed and verified. While future studies are required to also analyze the rest of the proposed mechanisms, the results clearly indicate that large savings come at only a small reduction of the security level. Therefore, we show that such reactive mechanisms that allow to trade-off security requirements and load might open the way to really practical approaches.

Still there is need for ongoing analysis as the reduced security level might open new attack vectors that we have only scratched in this paper.

\section{REFERENCES}

[1] E. Schoch, F. Kargl, T. Leinmüller, and M. Weber, "Communication Patterns in VANETs," IEEE Comm. Magazine, vol. 46, no. 11, Nov. 2008.

[2] P. Papadimitratos et al., "Secure Vehicular Communications: Design and Architecture," IEEE Comm. Magazine, vol. 46, no. 11, pp. 2-8, Nov. 2008.

[3] "IEEE P1609.2 - WAVE Standard for Security Services for Application and Management Services," Jun. 2006.

[4] G. Calandriello, P. Papadimitratos, J.-P. Hubaux, and A. Lioy, "Efficient and robust pseudonymous authentication in VANET," in ACM VANET '07. Sep. 2007.

[5] M. Raya and J.-P. Hubaux, "The Security of Vehicular Ad Hoc Networks," in SASN 2005, Nov. 2005.

[6] S. Peter, et al., "Flexible hardware reduction for elliptic curve cryptography in GF(2m)," in DATE 'O7. 2007.

[7] Y.-C. Hu and Kenneth P. Laberteaux, "Strong VANET Security on a Budget," in escar 2006, Nov. 2006.

[8] "IEEE 802.11 - Wireless LAN Medium Access Control (MAC) and Physical Layer (PHY) Specifications 2007", June 2007.

[9] Stephan Eichler, "Performance Evaluation of the IEEE 802.11p WAVE Communication Standard," in VTC Fall. 2007.

[10] T. Leinmüller, "Car2x Comm. - Challenges, Standardization and Implementation in Europe and in the US," in car2 $x$ Communication - opportunities and challenges, Nov. 2007.

[11] F. Kargl, E. Schoch, B. Wiedersheim, and T. Leinmüller, "Secure and Efficient Beaconing for Vehicular Networks (Short Paper)" in 5th ACM VANET 2008. September 2008.

[12] P. Papadimitratos, G. Calandriello, A. Lioy, and J.-P. Hubaux, "Impact of Vehicular Communication Security on Transportation Safety," in MOVE 2008, Apr. 2008.

[13] D. R. Choffnes and F. E. Bustamante, "An Integrated Mobility and Traffic Model for Vehicular Wireless Networks," in 2nd ACM VANET2005, Sep. 2005.

[14] R. Krüger et al., "Statistical Analysis of the FleetNet Highway Movement Patterns,", Department for Math. and Comp. Science, Univ. of Mannheim, TR-2005-004, 2005.

[15] R. Barr, Z. Haas, and R. van Renesse, "JiST: An efficient approach to simulation using virtual machines," in Software Practice \& Experience, vol. 35, no. 6, pp. 539-576, 2005. 